\title{
Finite element modeling to predict procedural success of thoracic endovascular aortic repair in type $A$ aortic dissection
}

\author{
Xun Yuan, MBBS, MMED, ${ }^{\mathrm{a}, \mathrm{b}}$ Xiaoxin Kan, BSc, MSc, ${ }^{\mathrm{c}} \mathrm{Xiao}$ Yun $\mathrm{Xu}, \mathrm{PhD},{ }^{\mathrm{c}}$ and \\ Christoph A. Nienaber, MD, $\mathrm{PhD}^{\mathrm{a}, \mathrm{b}}$
}

\begin{abstract}
Objective: Thoracic endovascular aortic repair (TEVAR) is recommended for type $B$ aortic dissection and recently has even been used in selected cases of proximal (Stanford type A) aortic dissections in scenarios of prohibitive surgical risk. However, mechanical interactions between the native aorta and stent-graft are poorly understood, as some cases ended in failure. The aim of this study is to explore and better understand biomechanical changes after TEVAR and predict the result via virtual stenting.
\end{abstract}

Methods: A case of type A aortic dissection was considered inoperable and selected for TEVAR. The procedure failed due to stent-graft migration even with precise deployment. A novel patient-specific virtual stent-graft deployment model based on finite element method was employed to analyze TEVAR-induced changes under such conditions. Two landing positions were simulated to investigate the reason for stent-graft migration immediately after TEVAR and explore options for optimization.

Results: Simulation of the actual procedure revealed that the proximal bare metal stent pushed the lamella into the false lumen and led to further stent-graft migration during the launch phase. An alternative landing position has reduced the local deformation of the dissection lamella and avoided stent-graft migration. Higher maximum principal stress $(>20 \mathrm{KPa})$ was found on the lamella with deployment at the actual position, while the alternative strategy would have reduced the stress to $<5 \mathrm{KPa}$.

Conclusions: Virtual stent-graft deployment simulation based on finite element model could be helpful to both predict outcomes of TEVAR and better plan future endovascular procedures. (JTCVS Techniques 2020;4:40-7)

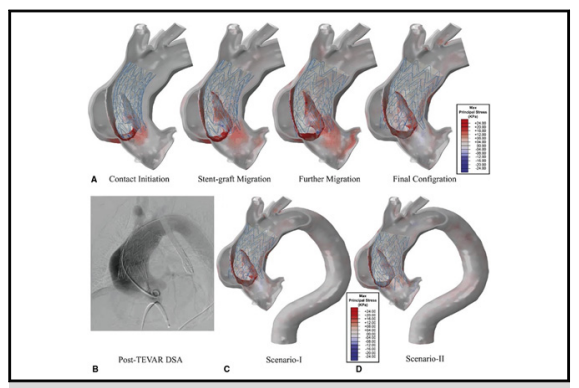

Virtual stent-graft deployment simulation to predict outcome of TEVAR.

CENTRAL MESSAGE

TEVAR in TAAD needs optimization to benefit more patients.

Virtual stent-graft deployment simulation can predict TEVARinduced biomechanical changes and assist intervention planning.

\section{PERSPECTIVE}

TEVAR in TAAD remains challenging due to anatomical complexity and device limitation. Image-based computational simulation of stentgraft deployment can potentially be used to predict the immediate outcome of TEVAR and optimize endovascular procedures in the future.

See Commentary on page 48.
Video clip is available online.

From the ${ }^{\mathrm{a} C a r d i o} \operatorname{logy}$ and Aortic Centre, Royal Brompton \& Harefield NHS Foundation Trust; and ${ }^{\mathrm{b}}$ National Heart and Lung Institute, School of Medicine and ${ }^{\mathrm{c}} \mathrm{De}-$ partment of Chemical Engineering, Imperial College London, London, United Kingdom.

The authors acknowledge the Lee Family Scholarship for the support of Xun Yuan and the China Scholarship Council for the support of Xiaoxin Kan.

Dr Yuan and Mr Kan contributed equally to this work.

Received for publication Oct 7, 2020; accepted for publication Oct 8, 2020; available ahead of print Oct 13, 2020.
Aortic dissection is a life-threatening condition with an incidence of 2.6 to 3.5 cases per 100,000 person-years. ${ }^{1,2}$ According to current guidelines, the standard care for proximal aortic dissection is surgery. However, even with modern techniques, perioperative mortality is $15 \%$ to $30 \%$ in

\footnotetext{
Address for reprints: Christoph A. Nienaber, MD, PhD, Cardiology and Aortic Centre, Royal Brompton \& Harefield NHS Foundation Trust, Imperial College London, London, SW3 6NP United Kingdom (E-mail: C.Nienaber@rbht.nhs.uk). 2666-2507

Copyright $(2020$ The Authors. Published by Elsevier Inc. on behalf of The American Association for Thoracic Surgery. This is an open access article under the CC BY-NCND license (http://creativecommons.org/licenses/by-nc-nd/4.0/). https://doi.org/10.1016/j.xjtc.2020.10.006
} 


\section{Abbreviations and Acronyms}

$\mathrm{CT}=$ computed tomography

$\mathrm{ECG}=$ electrocardiogram

TEVAR $=$ thoracic endovascular aortic repair

elderly patients with comorbidities. ${ }^{3}$ To date, endovascular strategies have emerged for managing various aortic pathologies, ${ }^{4,5}$ have been established for treatment of distal (Stanford type B) aortic dissections, and recently even been used in selected cases of proximal (Stanford type A) aortic dissections. ${ }^{6,7}$ Acute type A aortic dissection usually requires urgent replacement of the ascending aorta ${ }^{8-10}$; selected cases, however, may qualify for thoracic endovascular aortic repair (TEVAR) in scenarios of prohibitive surgical risk. ${ }^{11,12}$ TEVAR, where applicable, may potentially lower the procedural/in-hospital mortality risk, particularly as the technology improves. ${ }^{13}$ However, due to the complexity in anatomic structure and mechanical motion in the ascending aorta, there is currently no designated ascending aorta stentgraft available. ${ }^{14}$ In addition, interactions between the native aorta and stent-graft and resulting changes in aortic wall stress, hemodynamic, and aortic root motion are unknown.

Virtual stent-graft deployment simulation is a computational approach based on finite element method, which can mimic biomechanical interactions between stent-graft and aorta during a TEVAR procedure by using preinterventional computed tomography (CT) angiogram and provide quantitative prediction of stent-graft position and wall stress. ${ }^{15}$ Virtual stent-graft simulation models have been reported and applied to abdominal aortic aneurysm demonstrating accuracy and potential as a interventional planning tool. ${ }^{16,17}$ Similar approaches have been applied to type B aortic dissections in an attempt to identify factors leading to retrograde type A dissection following TEVAR. ${ }^{18}$ However, virtual stent-graft deployment simulation of TEVAR for proximal aortic dissection is challenging due to complex anatomical structure and nonlinear contact relationship between stent-graft and ascending aorta. So far, there is no published study of patient-specific virtual stent-graft deployment simulation in type A aortic dissection yet. We report a retrospective study of a failed TEVAR in DeBakey II dissection caused by subsequent migration postprocedure. The migration following stent-graft deployment was reproduced using a virtual simulation model in an attempt to understand why the procedure failed. An alternate deployment scenario was simulated to explore possibilities of avoiding migration.

\section{CASE REPORT}

An 80-year-old man was admitted with sudden onset of severe chest pain radiating to neck and jaw lasting for
13 hours after presenting to the emergency department of a district general hospital. He had a history of arterial hypertension, bilateral nephrolithiasis and reduced renal function, and a recent hernia repair. Electrocardiogram (ECG) showed rapid atrial fibrillation, right bundle branch block, and t-wave inversion on leads V2-V6, I, II, III, and aVF (Figure 1) reflecting left ventricular hypertrophy due to long standing hypertension. Troponin I had risen to $269 \mathrm{ng} / \mathrm{L}$ and then levelled off. A diagnostic CT angiogram revealed a proximal aortic dissection (DeBakey II), prompting transfer to intensive care unit and further on to a tertiary care aortic centre for surgery. Upon transfer, the patient developed pyrexia with episodes of rigor likely to be caused by infected venous leg ulcers that led to the decision to delay swift surgical repair and rather monitor the patient closely under broad spectrum antibiotic coverage, careful blood pressure adjustment and a reduced dose of $2.5-\mathrm{mg}$ bid apixaban.

A follow-up ECG-gated contrast-enhanced CT angiogram a week later showed stability of the confined dissection (DeBakey II) with no signs of progression, one single entry tear and no aortic valve dysfunction; coronaries were not compromised by the aortic dissection (Figure 2, A). In agreement with the patient and in view of both comorbidities and age, an operation was again deferred (EuroSCORE [European System for Cardiac Operative Risk Evaluation] II $47.86 \%$ and Society of Thoracic Surgeons score $53.694 \%$ ) while the patient stayed under close ambulatory surveillance. On the second follow-up scan 3 weeks later, expansion of the false lumen to $>60 \mathrm{~mm}$ was noted with no other concomitant changes. Subsequent multidisciplinary team decision recommended an endovascular approach with placement of a suitable compliant stentgraft to cover the single entry, as the distance between coronaries and the brachiocephalic branch was sufficient to accommodate a 45- $\times 100$-mm GORE C-TAG Active Control device (W. L. Gore \& Associates, Inc, Flagstaff, Ariz).

Thus, 43 days after the onset of the DeBakey II dissection, a TEVAR procedure was conducted with the patient under general anesthesia. Using femoral access and a 22F DrySeal introducer, we positioned a $45-\times 100-\mathrm{mm}$ GORE C-TAG Active Control precisely in the ascending aorta guided by fluoroscopy and transesophageal echocardiography to cover the tear without obstructing aortic sidebranches under low pressure conditions induced by rapid right ventricular pacing at $180 \mathrm{bpm}$. After successful launch of the semi-opened device under image overlay, the subsequent angiogram revealed that the stent-graft migrated seconds after deployment with its distal end being dislodged into the false lumen (Figure 2, B). Although hemodynamically stable, no second stent was placed in the absence of any landing zone left, in favor of swift elective surgery. The patient stayed in hospital as he developed intermittent complete heart block requiring dual chamber pacing in 


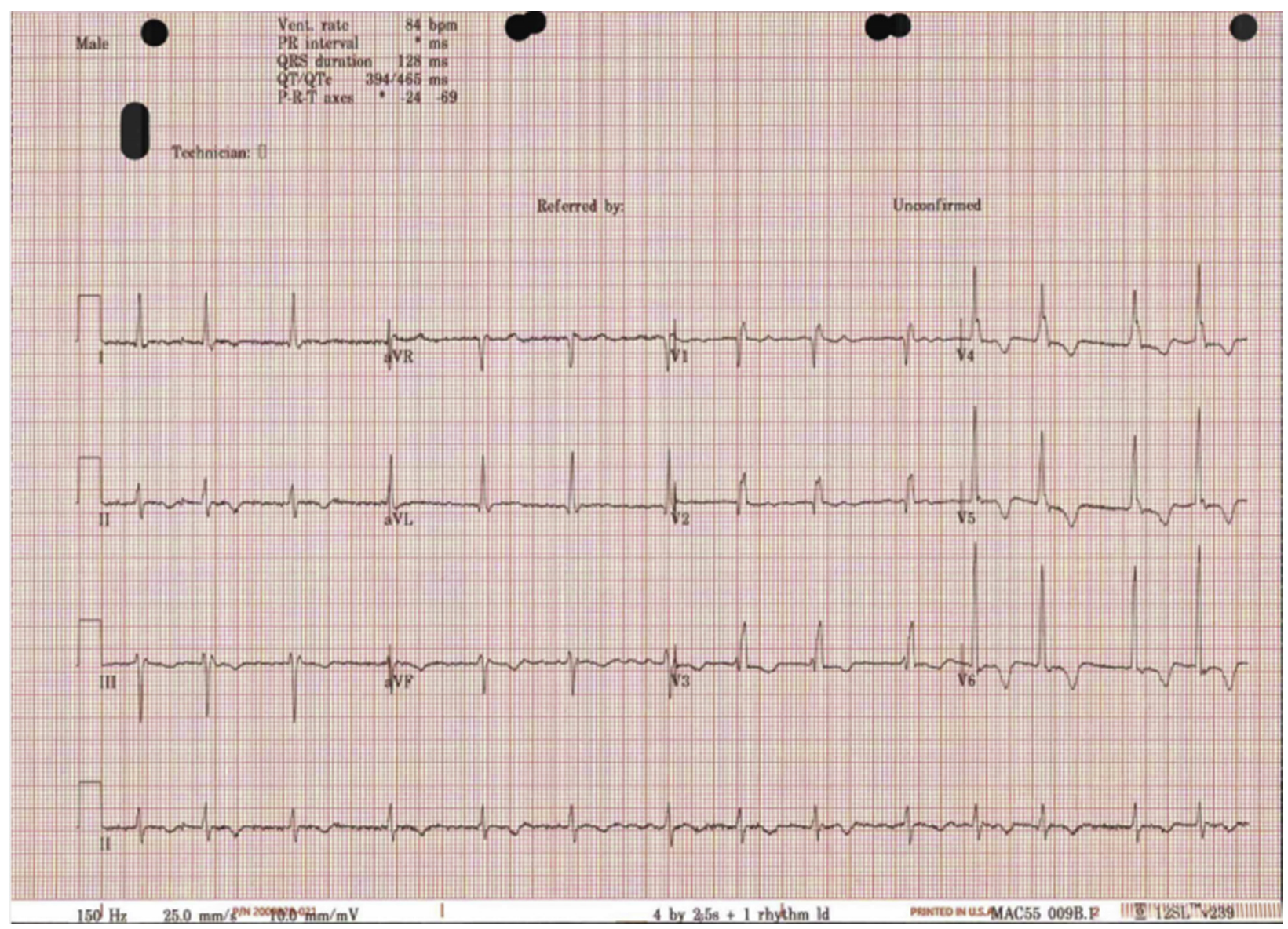

FIGURE 1. Electrocardiogram at admission shows rapid atrial fibrillation, right bundle branch block, and t-wave inversion on leads V2-V6, I, II, III, and aVF, reflecting left ventricular hypertrophy due to long standing hypertension.

DDD mode. One week later, successful surgery was performed by removing the stent-graft under conditions of $28^{\circ} \mathrm{C}$ hypothermia and antegrade brain perfusion and replacing the ascending aorta/hemiarch with a $32-\mathrm{mm}$ Gelweave graft (Vascutek Terumo, Scotland, United Kingdom) with sidearm. The postoperative course was complicated by both temporary respiratory and kidney failure, requiring prolonged ventilation and intermittent renalreplacement therapy, eventually leading to full recovery on oral diuretics and anticoagulation.

Next-generation deoxyribonucleic acid sequencing and bioinformatic copy number analysis of a 62 gene subpanel associated with aortopathy and connective tissue disorders failed to reveal any pathogenic sequence variants or copy number changes.

\section{METHODS AND RESULTS}

\section{Virtual Stent-Graft Deployment Simulation}

A detailed biomechanical analysis of the TEVAR procedure was performed using a virtual stent-graft deployment simulation model based on the finite element method. ${ }^{19}$ The preinterventional CT angiogram images were processed to reconstruct the 3-dimensional patientspecific aortic geometry and the dissection lamella. Ethical approval was obtained from local Ethics Committee, and written consent was given by the patient. The aortic wall was assumed to have a uniform thickness of $1.5 \mathrm{~mm}$ and its mechanical behavior was described using second ordered Ogden hyperelastic model fitted to the circumferential tensile test data of aortic tissue, ${ }^{20}$ whereas the dissection lamellar was assumed to have linear elastic material properties with non-uniform thickness as extracted from ECG-gated CT angiographic images. Details of the constitutive models and mechanical properties are provided in Table 1. The preinterventional aorta model was meshed using C3D4 elements for the virtual stent-graft simulation. The proximal end and distal ends of the aorta model were fixed in all directions.

The stent-graft model was created by following the design specification of the device $(45-\times 100-m m$ GORE C-TAG Active Control) used in the TEVAR procedure consisting of 2 components: a nitinol metallic stent and e-polytetrafluoroethylene fabric. A mathematical equation describing the geometry of the metallic stent skeleton was 


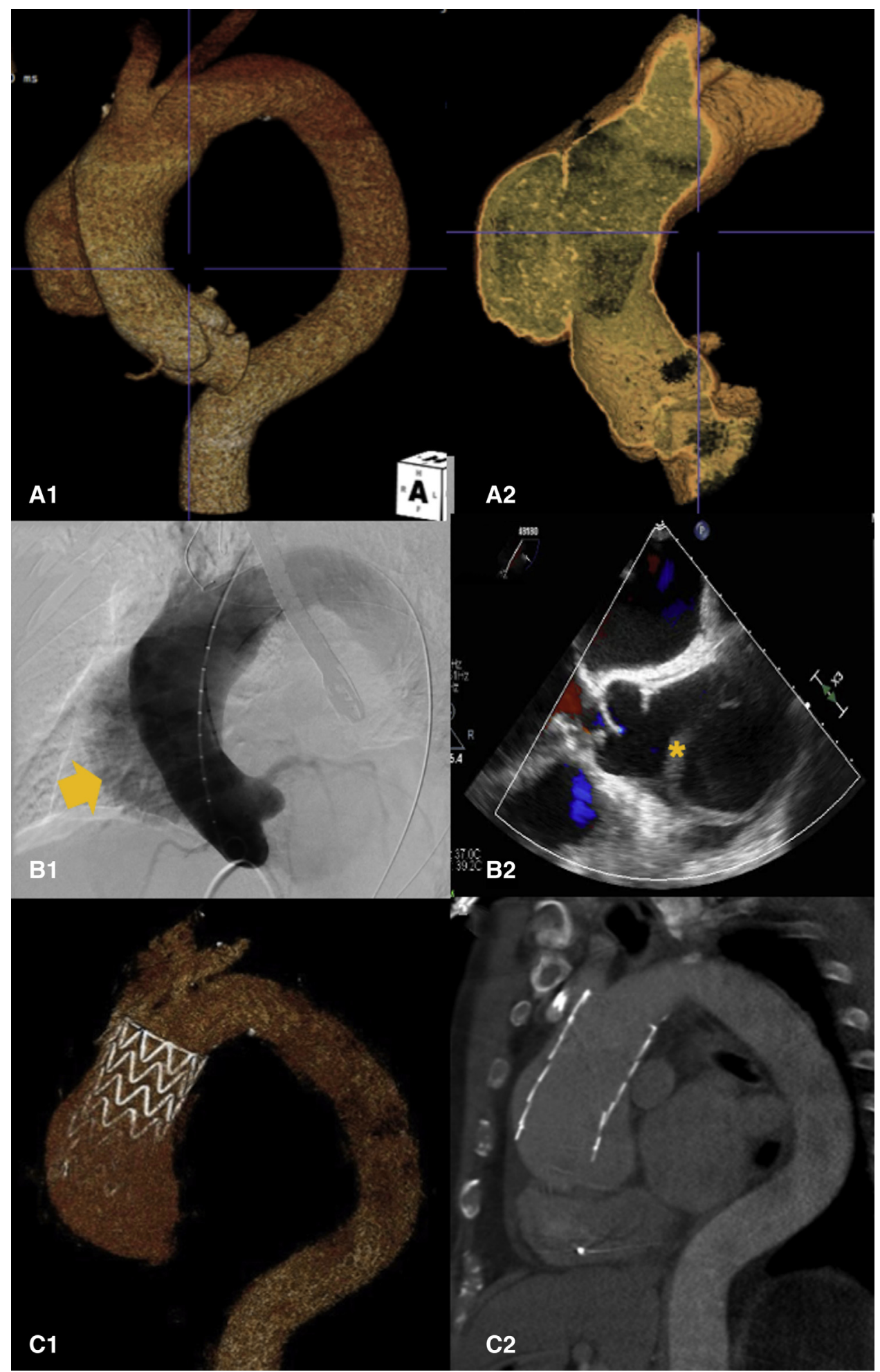

FIGURE 2. Periprocedural images of an inoperable patient with type A aortic dissection managed by thoracic endovascular aortic repair. A, 3-dimentional reconstruction of a computed tomographic angiogram with type A aortic dissection showing a single large entry tear; B1, true and false lumens opacified using digital subtraction angiography; B2, transesophageal echocardiogram showing true and false lumen and the lamella (asterisk); C1, postintervention 3-dimentional computed tomographic angiogram showing stent-graft migration and the proximal part of stent-graft pushing into the false lumen best seen on the 2-dimentional image $(\mathrm{C} 2)$. 
TABLE 1. Material parameters and constitutive law used in the virtual stent-graft simulation model

\begin{tabular}{ll}
\hline \multicolumn{1}{c}{ Part name } & \multicolumn{1}{c}{ Material property } \\
\hline Aortic wall & Second-ordered Ogden model \\
& $W=\sum_{i=1}^{2} \frac{2 \mu_{i}}{\alpha_{i}^{2}}\left(\lambda_{1}^{-\alpha_{i}}+\lambda_{2}^{-\alpha_{i}}+\lambda_{3}^{-\alpha_{i}}-3\right)$ \\
& $\mu_{1}=1.274 \mathrm{MPa}, \alpha_{1}=24.074$, \\
& $\mu_{2}=-1.211 \mathrm{MPa}, \alpha_{2}=24.073$ \\
Dissection lamella & Young's modulus: $\mathrm{E}_{\text {lamellar }}=277 \mathrm{KPa}$ \\
& Poisson's ratio: $\nu=0.49$ \\
Metallic stent & Young's modulus (austenite): \\
& E stent $=51,700 \mathrm{MPa}$ \\
Graft & Poisson's ratio: $\nu=0.3$ \\
& Young's modulus: $\mathrm{E}_{\text {graft }}=55.2 \mathrm{MPa}$ \\
\hline
\end{tabular}

developed based on the detailed dimensions of the stentgraft device (Figure 3,C). While the fully crimped state of the stent-graft was not simulated, the stent skeleton was assumed to have elastic material properties in austenite phase at body temperature, ${ }^{15}$ whereas the stent-graft fabric was modeled as a cylindrical tube with a thickness of $0.1 \mathrm{~mm}$ with linear elastic material properties. ${ }^{21}$ The metallic stent and stent-graft fabric were assembled together according to specific design considerations.

The initial geometric configuration used in the computational simulation was based on the preinterventional CT angiogram, whereas the stent-graft positioning was based on the angiographic image during the TEVAR procedure. Simulation of stent-graft deployment was controlled by the deformation of a virtual catheter; the virtual stent- graft was crimped from its stress-free state to the diameter of the delivery catheter. Both proximal landing position and assumed curvature of the virtual stent-graft were simulated before removing the virtual catheter. The virtual stent-graft landing position was selected from the fluoroscopic image acquired at semi-open stage before TEVAR launch, as shown in Figure 3, A. Two different landing positions were simulation. Scenario I corresponds to the position adopted in the actual procedure. To understand the influence of stent-graft proximal landing position on its biomechanical performance, scenario II was simulated where the landing position was moved toward the aortic root by $10 \mathrm{~mm}$ (Figure 3, B). Simulation results were verified by comparing the simulated stent-graft position with the real stent-graft position shown in the digital subtraction angiography after stent-graft launch.

The virtual stent-graft deployment simulation was performed with ABAQUS explicit solver (Dassault Systèmes, France). All contacts were modeled by using the general penalty contact with a friction coefficient of 0.2 under the Coulomb friction law. ${ }^{21}$ The ratio of the kinetic energy and internal energy (ALLKE/ALLIE) was kept below $10 \%$ to ensure that the simulation was quasi-static. The simulation took 30 hours for each scenario using a workstation with Duo Intel Xeon E5-2630 central processing units (40 cores in total).

\section{Patient-Specific Simulation Results}

The simulation results of scenario I are shown in Figure 4, $A$. The maximum principal stresses to the aortic

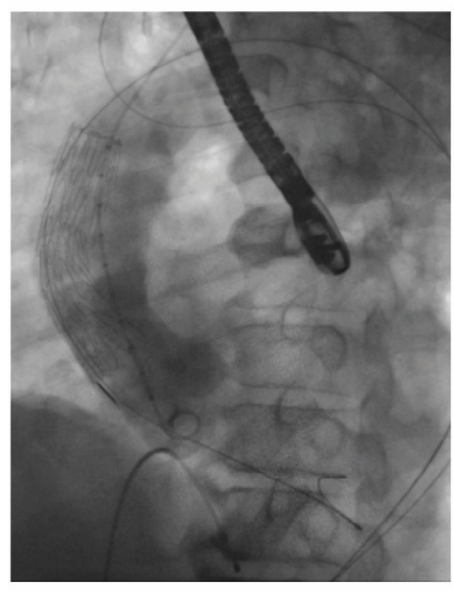

A

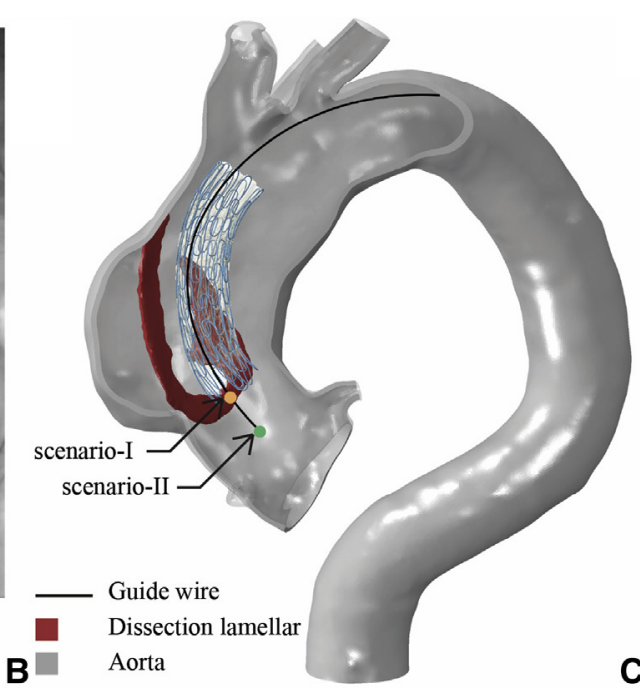

FIGURE 3. Patient-specific finite element model analysis. A, Fluoroscopic view of stent-graft positioning in semi-open stage before launch. B, Simulated image of the stent-graft in the ascending aorta with virtual adaptation to the aortic curvature. The orange circle marks the proximal landing position in scenario I, which simulates the actual thoracic endovascular aortic repair procedure, whereas the green circle simulates the proximal position of stent-graft in scenario II, which assumed a more proximal positioning by $10 \mathrm{~mm}$. C, The mathematical equation governed stent-graft geometry was created by following the design details of the stent-graft device. The blue wire represents the nitinol metallic skeleton, whereas the yellow tube represents the graft fabric. $e$-PTFE, Expanded polytetrafluoroethylene. 


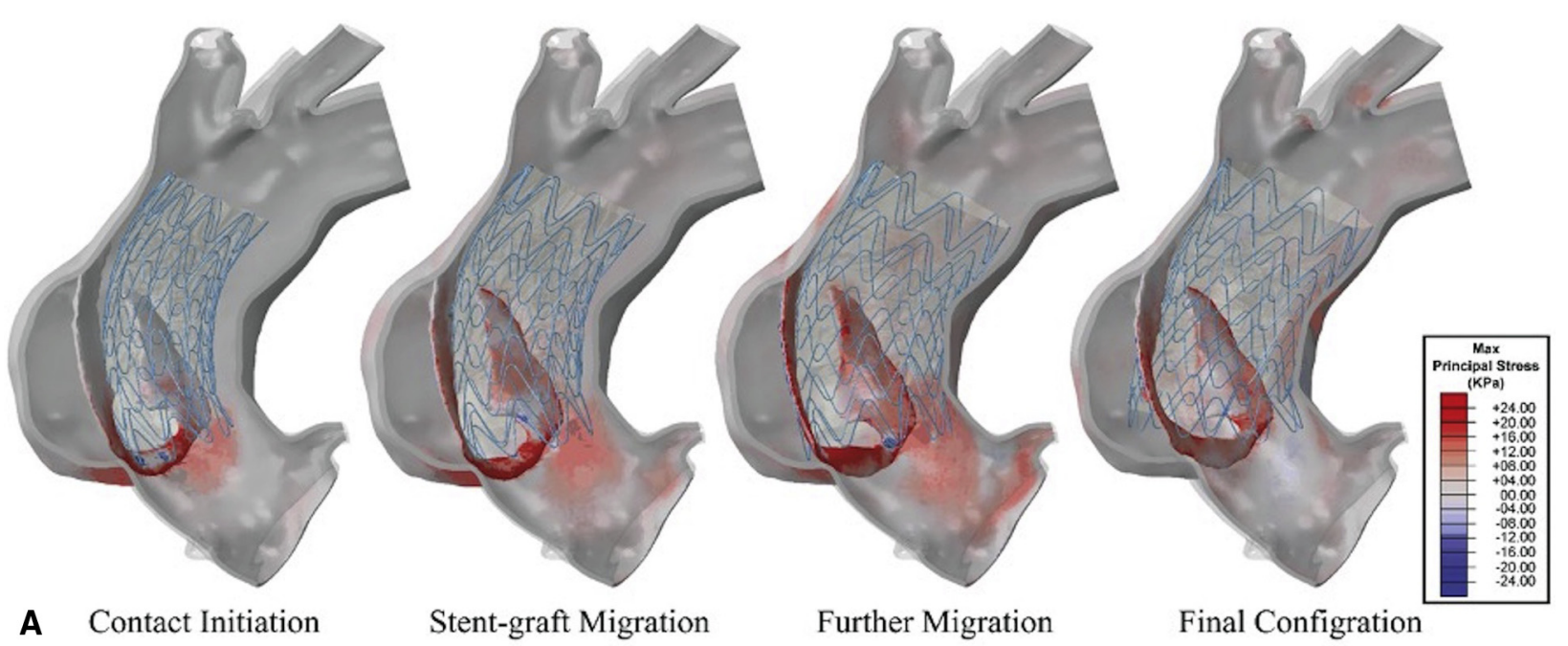

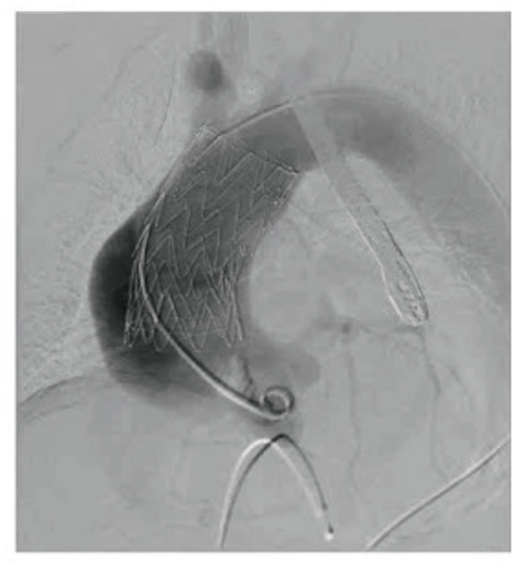

Post-TEVAR DSA
B

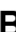

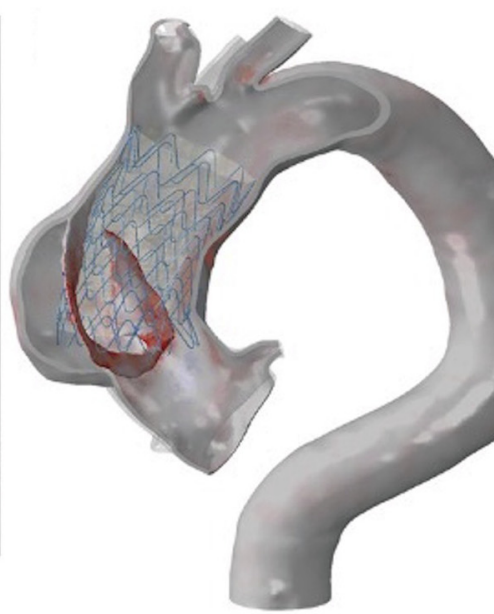

Scenario-I

C

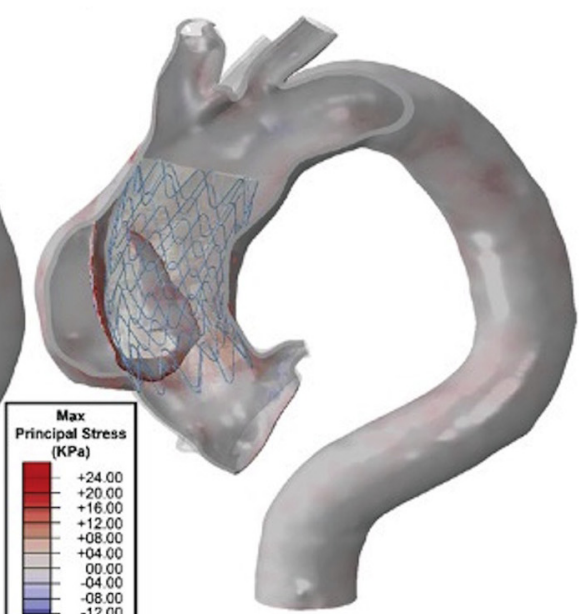

Scenario-II

FIGURE 4. Virtual stenting simulation results. A, Simulated description of various phases of stent-graft placement from left to right. B, Postinterventional digital subtraction angiogram showing stent-graft migration. C, Virtual stent-graft deployment simulation of the actual procedure (scenario I). D, Virtual stent-graft deployment simulation based on the assumption of stent-graft deployed 10 mm proximally (scenario II). The final configuration of stentgraft in both scenarios are projected onto the postinterventional digital subtraction angiogram.

wall and dissection lamella are displayed to analyze stress redistribution during stent-graft deployment, where positive and negative values correspond to tension and compression, respectively. When the deployment was initiated, contact between the proximal bare metal stent apexes and the lamella led to local stress concentration (blue spot in Figure 4, A), causing the dissection lamella to deform toward the false lumen. This local deformation allowed the proximal bare metal stent to further push into the false lumen, as illustrated by the instantaneous snapshots captured during simulation (Video 1). After the interaction reached a stable state, the final stent-graft position obtained from the scenario I simulation (Figure 4, C) was compared with digital subtraction angiography acquired after stent- graft deployment (Figure 4, B) demonstrating congruence. Minor discrepancies were observed at the distal end of stent-graft, which was likely caused by further stent-graft migration under blood flow.

Results from the scenario II simulation showed less deformation of stent-graft toward the false lumen and suggested that the proximal end of the stent-graft would not be pushed into the false lumen (Figure 4, D, Video 1). Comparison of the maximum principal stresses between both simulated scenarios revealed that the dissection lamella was exposed to lower stress in scenario II than in scenario I. High stresses $(>20 \mathrm{KPa})$ were observed at the entry tear of the lamella in scenario I, whereas scenario II simulation revealed a maximum stress of $5 \mathrm{KPa}$ at the same location. 


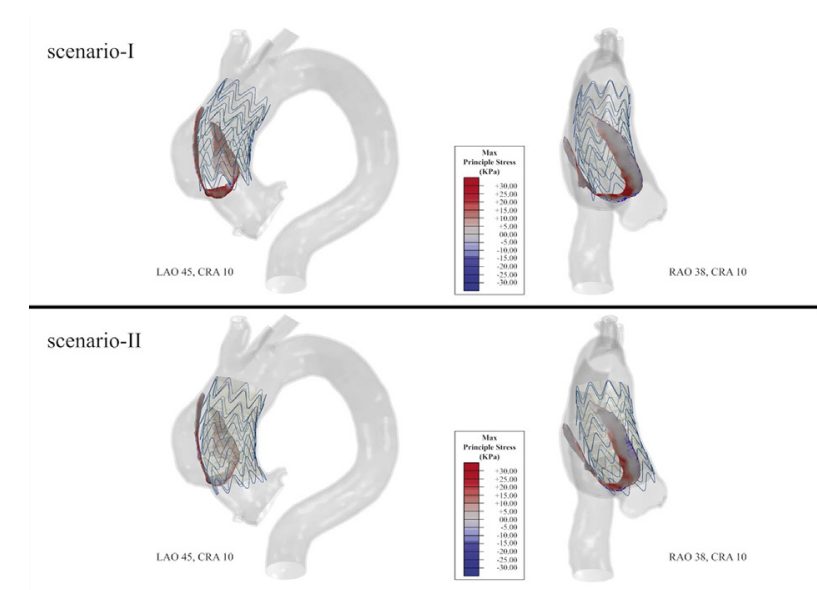

VIDEO 1. Computational virtual stent-graft deployment simulation performed by using finite element analysis. Scenario I was based on the preTEVAR images and the simulation accurately predicted the actual result. The proximal bare metal stent pushed the lamella into the false lumen and led to further stent-graft migration during the launching phase. Scenario II was based on the assumption that stent-graft would be deployed $10 \mathrm{~mm}$ proximally and close to the aortic root. In addition, the simulated tissue stress maps revealed differences in the lamella with lower focal stress in scenario II compared with scenario I. This might explain the failure in this case (high stress combined with localized deformation caused stentgraft migration during TEVAR). Video available at: https://www.jtcvs. org/article/S2666-2507(20)30575-7/fulltext.

\section{DISCUSSION}

Although endovascular procedures have recently been applied with some success even to the ascending aorta in various chronic scenarios, ${ }^{6}$ the technology appears not ready for prime time for a number of reasons: first, there are no dedicated endovascular devices available for specific use in the ascending aorta addressing the challenges of the anatomy and the 3-dimensional motion of the ascending aorta; second, current devices should precisely fit between coronary ostia and the brachycephalic trunk; and third, the delivery of such device must be controlled and precise to avoid major problems.

In our highly selective case such pre-conditions seem to be fairly well met, given the contraindication for open surgery, the suitable anatomy for a flexible device (45$\times$ 100-mm GORE C-TAG Active Control), and the modern automatic release mechanisms supported by rapid right ventricular pacing and low blood pressure for precise placement. Nevertheless, the procedure failed as well documented in Figure 4, $B$. The important questions are now how and whether this could have been predicted.

To explore the outcome before clinical application finite element modelling of the interaction between an endovascular device and surrounding anatomy was brought in. Based on preinterventional CT angiogram images, virtual stent-graft deployment simulation performed offline revealed interesting findings. The crown, proximal bare metal stent was found to push against the dissection lamella at the level of the proximal landing zone (Figure 4,C) with high focal stresses in the tissue. High compression stress and localized deformation of the dissecting lamella induced by radial force of the stent-graft caused its migration during the launching phase. The localized deformation further reduced local contact pressure and friction between the stent-graft and the dissecting lamella allowing the stentgraft to slide on the lamella. After full deployment, high local stress was still present around the entry tear facilitating the stent-graft to straighten after deployment as shown by the mismatch between the digital subtraction angiography in the follow-up CT angiogram (Figures 2, $C$, and $4, B$ ). Our simulation suggested that the proximal landing zone was too close to the tear and the tissue too vulnerable to support the radial force of a self-expanding stent-graft.

By moving the proximal landing position about $10 \mathrm{~mm}$ toward the aortic valve as simulated in scenario II, the predicted stress level in the dissecting lamella was much lower, avoiding overstretching the dissection lamella and stentgraft migration as predicted by the scenario I simulation. Simulation of scenario II suggested the better strategy to prevent stent-graft migration. Localized deformation and stress concentration were reduced which would have avoided migration and dislocation of the stent into the false lumen. Meanwhile, the scenario II strategy reduced the risk of further migration by decreasing the principal stress level around the tear from $20 \mathrm{KPa}$ (in scenario I) to $<5 \mathrm{KPa}$.

The remarkable agreement between the simulation results of scenario I and the clinical outcome is promising and suggests that our virtual stent-graft deployment simulation method has the potential to not only predict changes in biomechanical conditions induced by TEVAR but also to improve planning of TEVAR procedure in the future. The potential value of virtual stent-graft deployment simulation has been well illustrated by this case study, demonstrating that slight differences in stent-graft landing position can determine the outcome of a given procedure.

In summary, biomechanical interactions between stentgraft and native aorta play a vital role in determining the immediate result of stent-graft deployment. These interactions cannot be directly evaluated from anatomic dimensions. Our virtual stent-graft deployment model has provided new insights into the stent-graft behavior within dissected aorta and successfully reproduced the immediate outcome of TEVAR as observed in vivo; the model indicated that TEVAR-induced changes in local biomechanical conditions were responsible for stent-graft migration during the launching phase. A modified deployment position was simulated showing a lower risk of migration with improvement in the local biomechanical environment. 


\section{Limitations}

The virtual stent-graft deployment simulation involves several assumptions. These include a uniform wall thickness and the use of an isotropic model for the aortic tissue. Furthermore, the simulation was based on static images neglecting the 3-dimensional motion of the aortic root and its impact on interaction between the stent-graft and the aorta. In addition, the initial patient-specific aorta model geometry was reconstructed from CT angiogram images, representing the configuration of the aorta under internal blood pressure. However, the zero-stress configuration of aortic tissue and pre-stress of the aorta were not considered in this simulation model. The influence of pulsatile blood flow and pulsating pressure was also neglected. Finally, the mechanical properties of aortic tissue in the setting of dissection are based on data in the literature rather than patient-specific. Therefore, further improvement of the virtual stent-graft deployment model and thorough validation of the simulation results are needed before adoption for real clinical scenarios.

\section{CONCLUSIONS}

The virtual stent-graft deployment simulation has shown potential in predicting the immediate outcome of TEVAR deployment by analyzing TEVAR-induced changes under given biomechanical conditions in a patient-specific manner. High stress concentration combined with localized deformation was responsible for stent-graft migration observed during TEVAR. As a potential tool for future interventional planning and optimization the concept needs further validation in a prospective clinical setting.

\section{Conflict of Interest Statement}

The authors reported no conflicts of interest.

The Journal policy requires editors and reviewers to disclose conflicts of interest and to decline handling or reviewing manuscripts for which they may have a conflict of interest. The editors and reviewers of this article have no conflicts of interest.

\section{References}

1. Olsson C, Thelin S, Stahle E, Ekbom A, Granath F. Thoracic aortic aneurysm and dissection: increasing prevalence and improved outcomes reported in a nationwide population-based study of more than 14,000 cases from 1987 to 2002. Circulation. 2006:114:2611-8.

2. Yuan X, Mitsis A, Tang Y, Nienaber CA. The IRAD and beyond: what have we unravelled so far? Gen Thorac Cardiovasc Surg. 2019;67:146-53.

3. Trimarchi S, Eagle KA, Nienaber CA, Rampoldi V, Jonker FH, De Vincentiis C, et al. Role of age in acute type A aortic dissection outcome: report from the international registry of acute aortic dissection (IRAD). J Thorac Cardiovasc Surg. 2010;140:784-9.
4. Erbel R, Aboyans V, Boileau C, Bossone E, Bartolomeo RD, Eggebrecht H, et al. 2014 ESC guidelines on the diagnosis and treatment of aortic diseases: document covering acute and chronic aortic diseases of the thoracic and abdominal aorta of the adult. The task force for the diagnosis and treatment of aortic diseases of the European Society of Cardiology (ESC). Eur Heart J. 2014;35:2873-926.

5. Riambau V, Bockler D, Brunkwall J, Cao P, Chiesa R, Coppi G, et al. Editor's choice-management of descending thoracic aorta diseases: clinical practice guidelines of the European Society for Vascular Surgery (ESVS). Eur J Vasc Endovasc Surg. 2017;53:4-52.

6. Nienaber CA, Sakalihasan N, Clough RE, Aboukoura M, Mancuso E, Yeh JS, et al. Thoracic endovascular aortic repair (TEVAR) in proximal (type A) aortic dissection: ready for a broader application? J Thorac Cardiovasc Surg. 2017; 153:S3-11.

7. Roselli EE, Idrees JJ, Johnston DR, Roselli EE, Idrees JJ, Johnston DR, et al. Zone zero thoracic endovascular aortic repair: a proposed modification to the classification of landing zones. J Thorac Cardiovasc Surg. 2018;155:1381-9.

8. Santini F, Montalbano G, Casali G, Messina A, Iafrancesco M, Luciani GB, et al Clinical presentation is the main predictor of in-hospital death for patients with acute type A aortic dissection admitted for surgical treatment: a 25 years experience. Int J Cardiol. 2007;115:305-11.

9. Nienaber C, Rosendahl U, Yuan X. What is the strategy for strategic arch resection in acute proximal aortic dissection? J Thorac Dis. 2020;12:3418-21.

10. Yuan X, Mitsis A, Ghonem M, Iakovakis I, Nienaber CA. Conservative management versus endovascular or open surgery in the spectrum of type B aortic dissection. J Vis Surg. 2018;4:59.

11. Kreibich M, Soekeland T, Beyersdorf F, Bavaria JE, Schrofel H, Czerny M, et al Anatomic feasibility of an endovascular valve-carrying conduit for the treatment of type A aortic dissection. J Thorac Cardiovasc Surg. 2019;157: 26-34.e1.

12. Roselli EE, Hasan SM, Idrees JJ, Aftab M, Eagleton MJ, Menon V, et al. Inoperable patients with acute type A dissection: are they candidates for endovascular repair? Interact Cardiovasc Thorac Surg. 2017;25:582-8.

13. Lu Q, Liu L, Chang G, Chen X, Feng H, Zhang X, et al. Mid-term outcomes from a multicenter study: is TEVAR safe for ascending aortic dissection? Int J Cardiol. 2018;265:218-22.

14. Ma T, Dong ZH, Fu WG, Guo DQ, Xu X, Chen B, et al. Incidence and risk factors for retrograde type A dissection and stent graft-induced new entry after thoracic endovascular aortic repair. J Vasc Surg. 2018;67:1026-33.e2.

15. Derycke L, Perrin D, Cochennec F, Albertini JN, Avril S. Predictive numerical simulations of double branch stent-graft deployment in an aortic arch aneurysm. Ann Biomed Eng. 2019;47:1051-62.

16. Perrin D, Badel P, Orgeas L, Geindreau C, Dumenil A, Albertini JN, et al. Patientspecific numerical simulation of stent-graft deployment: validation on three clinical cases. J Biomech. 2015;48:1868-75.

17. Derycke L, Senemaud J, Perrin D, Avril S, Desgranges P, Albertini JN, et al Patient specific computer modelling for automated sizing of fenestrated stent grafts. Eur J Vasc Endovasc Surg. 2020;59:237-46.

18. Ma T, Dong ZH, Wang S, Meng ZY, Chen YY, Fu WG. Computational investigation of interaction between stent graft and aorta in retrograde type A dissection after thoracic endovascular aortic repair for type B aortic dissection. J Vasc Surg. 2018;68:14S-21S.e2

19. Romarowski RM, Conti M, Morganti S, Grassi V, Marrocco-Trischitta MM Trimarchi S, et al. Computational simulation of TEVAR in the ascending aorta for optimal endograft selection: a patient-specific case study. Comput Biol Med. 2018;103:140-7.

20. Deplano V, Boufi M, Gariboldi V, Loundou AD, D'Journo XB, Cautela J, et al Mechanical characterisation of human ascending aorta dissection. $J$ Biomech 2019;94:138-46

21. Desyatova A, MacTaggart J, Kamenskiy A. Effects of longitudinal pre-stretch on the mechanics of human aorta before and after thoracic endovascular aortic repair (TEVAR) in trauma patients. Biomech Model Mechanobiol. 2020;19:401-13.

Key Words: TEVAR, type A aortic dissection, virtual stentgraft deployment, finite element analysis 